Check for updates

Cite this: RSC Adv., 2018, 8, 31048

\title{
Effects of temperature on the fracture and fatigue damage of temperature sensitive hydrogels
}

\author{
Ni Zhang, Zhouzhou Pan, Jincheng Lei and Zishun Liu (D)* \\ As an excellent model material for fundamental studies on temperature-sensitive hydrogels, $\operatorname{poly}(N-$ \\ isopropylacrylamide) (NIPA) hydrogel has been applied in drug delivery, tissue engineering, and soft \\ robotics. However, the lack of study on fracture and fatigue hinders further development of hydrogels for \\ applications where cyclic loading-unloading is unavoidable. In this study, the fracture and fatigue damage \\ of the NIPA hydrogel were studied for the first time by pure shear tests at different temperatures. Fracture \\ behaviors were investigated under monotonic load from $31^{\circ} \mathrm{C}$ to $39^{\circ} \mathrm{C}$. It is found that the fracture energy \\ increases with the increase in temperature. The fracture energy is approximately $20 \mathrm{~J} \mathrm{~m}^{-2}$ near the volume \\ phase transition temperature. Temperature also significantly influences the fatigue life. By fitting the \\ experimental data, the fatigue limit $\lambda_{f}$ is determined. The results obtained from the experimental tests \\ would be important for the engineering applications of the NIPA hydrogels.
}

Received 2nd August 2018

Accepted 20th August 2018

DOI: $10.1039 / \mathrm{c} 8 \mathrm{ra06518g}$

rsc.li/rsc-advances

network hydrogels. They observed that internal damage accumulated over thousands of cycles until a steady state was reached under cyclic stretches for a sample without a pre-cut crack. For a sample with a pre-cut crack, the crack propagated over several cycles if the maximum stretch was larger than a critical value. Furthermore, the crack would not propagate over several cycles if the energy release rate was below a threshold, around $200-400 \mathrm{~J} \mathrm{~m}^{-2}$, which varied with the concentration of PAAM. Bai et al. $(2018)^{17}$ also studied the fatigue fracture of self-recovery hydrogels. They found that the stress-stretch curve recovered after thousands of cyclic stretches for a sample without a pre-cut crack. The covalent network played a critical role in determining the threshold of fatigue fractures, while the noncovalent interactions slowed down the extension of the crack under cyclic loads. Zhang et al. $(2018)^{18}$ studied the effect of water on the fatigue fracture of nearly elastic hydrogels by choosing the polyacrylamide hydrogel as a model material. They found that the water content largely influenced the fracture energy and fatigue thresholds.

In the aforementioned studies, the fatigue fracture for several types of hydrogels has been studied by pure shear tests, and the energy release rate was characterized as a function of the extension of crack length per cycle. In addition, the fatigue fracture threshold for corresponding hydrogels was obtained. However, all the existing researches concentrated on the fatigue fracture of hydrogels, while few studies focused on the fatigue damage of hydrogels. The effects of temperature on the fracture and fatigue of hydrogels remain unexplored. The lack of such a study hinders the further development of applications of hydrogels, particularly in fields that require their longevity under cyclic load conditions. 
In this paper, we focused on the study of the fracture and fatigue behaviours of NIPA hydrogels by pure shear tests and tried to reveal the effects of temperature on the fracture and fatigue mechanism of temperature-sensitive hydrogels. Fracture behaviours were investigated under monotonic load from $31{ }^{\circ} \mathrm{C}$ to $39^{\circ} \mathrm{C}$. It was found that the fracture energy increases with the increase in temperature. The fracture energy was approximately $20 \mathrm{~J} \mathrm{~m}^{-2}$ near the volume phase transition temperature. Temperature also significantly influenced the fatigue life. By fitting the experimental data, the fatigue limit $\left(\lambda_{\mathrm{f}}\right)$ was determined. The findings on the fracture and fatigue of temperature-sensitive hydrogels would provide some guidelines for possible applications in practical engineering.

\section{Experiments}

Thus far, there are many types of temperature-sensitive hydrogels that can be synthesized successfully, such as poly( $N$-isopropylacrylamide) (NIPA), methyl vinyl ether (MVE), and methacrylic acid (MAAC). Among those hydrogels, NIPA is the most excellent model material for fundamental studies on the temperature sensitivity of hydrogels. Furthermore, the volume phase transition temperature (VPTT) lies between $30{ }^{\circ} \mathrm{C}$ and $35{ }^{\circ} \mathrm{C}$ from experimental studies. The exact temperature is a function of the detailed microstructure of the macromolecule. ${ }^{19,20}$ Above the VPTT, the hydrogel is collapsed and hydrophobic, while it is swollen and hydrophilic below the VPTT. In this study, we selected NIPA hydrogel as a model material to study the fracture and fatigue damage of temperature sensitive hydrogels. It was synthesized via a free radical copolymerization, and the synthetic scheme was a modified scheme of Suzuki and Ishii. ${ }^{21}$

\section{Sample preparation}

We chose $N$-isopropylacrylamide (NIPA, monomer), $N, N^{\prime}$ methylenebisacrylamide (BIS, crosslinker), $N, N, N^{\prime}, N^{\prime}$-tetramethylethylenediamine (TEMED, accelerator) and ammonium persulfate (APS, initiator) as the main precursors. The detailed preparation process is shown as follows. (1) Initially, $3.9 \mathrm{~g}$ of purified NIPA monomer was dissolved in $50 \mathrm{~g}$ deionized water with $66.5 \mathrm{mg} N, N^{\prime}$-methylenebisacrylamide as the crosslinker. (2) The as-prepared solution was refrigerated at $4{ }^{\circ} \mathrm{C}$ for 8 hours to dissolve sufficiently. (3) Then, $120 \mu \mathrm{L}$ of TEMED was added as an accelerator into the solution. (4) Furthermore, $20 \mathrm{mg}$ of ammonium persulfate (APS) was added as an initiator for the condensation reaction. (5) The product was injected into a clean glass mould with $1 \mathrm{~mm}$ silicon spacer. In order to prevent the NIPA monomer from being separated at room temperature, the injection process was performed as quickly as possible. (6) The mould was refrigerated at $4{ }^{\circ} \mathrm{C}$, and the gelation process was performed for more than 24 hours for sufficient gelation. (7) The NIPA gel sheet was carefully removed from the glass mould and washed using deionized water to get rid of residual chemicals and unreacted monomers. (8) The prepared gel samples were immersed in deionized water at room temperature for 7 days to reach the equilibrium swollen state.
The VPTT test of the self-prepared NIPA hydrogel shows the VPTT ranging from $34.5{ }^{\circ} \mathrm{C}$ to $35.2^{\circ} \mathrm{C}$. Because of the hysteresis of swelling-deswelling, the VPTT from the heating process slightly differs with that from the heating process. Moreover, the slightly fluctuated VPTT value is attributed to mechanical constraints. $^{22}$

\section{Pure shear test}

For pure shear tests, the fully saturated samples were machined into rectangular sheets with dimensions of $5.0 \mathrm{~cm} \times 3.5 \mathrm{~cm}$ using a laser cutting machine. One type of obtained samples was used to test strain energy density and fatigue life, while the other samples with a $0.5 \mathrm{~cm}$-crack in the middle edge were used to test the critical stretch and calculate fracture energy (Fig. 1).

In the pure shear test, the tensile tester (SHIMADZU AGS-X, Shimadzu Corporation, Japan) with a load cell of $50 \mathrm{~N}$ was used for all of the tests. The lower side of the sample was fixed on the bottom of the water bath and the upper side was clamped using the metallic grippers of the tensile tester. To prevent the specimen from breaking or sliding during the tension process, the lower and upper sides of the gel were attached with ribbon gauze. The valid length $(H)$ of the sample between the fixed bottom and the upper gripper was set as $1.0 \mathrm{~cm}$. Under monotonic load, the samples are loaded until rupture in the water bath with a strain rate of $5 \mathrm{~mm} \mathrm{~min}^{-1}$ for each temperature (stepwise from $31^{\circ} \mathrm{C}$ to $39^{\circ} \mathrm{C}$ with $2{ }^{\circ} \mathrm{C}$ increments).

Due to the fact that the stress change is quite small when compared with the strain change after the yielding of the hydrogel, it is more reasonable to use strain (or stretch) as the control variable for the fatigue property tests. Therefore, in this study, stretch was adopted to study the fatigue of the NIPA hydrogel. For each test under different maximum stretch $\left(\lambda_{\max }\right)$, the number of cycles was increased until the sample ruptured. Fatigue life is defined as the maximum cycles corresponding to $\lambda_{\max }$. Considering no distinct rate dependence for the NIPA hydroge $^{22}$ and the time-consuming process of the cyclic tests, a larger loading strain rate of $30 \mathrm{~mm} \mathrm{~min}^{-1}$ was used in the mechanical cyclic tests. Fatigue tests were conducted at $31^{\circ} \mathrm{C}$ and $37{ }^{\circ} \mathrm{C}$ to show the fatigue damage below the VPTT and above the VPTT, respectively.

\section{Fracture behaviours}

To calculate the fracture energy of the NIPA hydrogel, the stress-stretch curves of samples with/without the pre-cut crack were measured at different temperatures through the VPTT by
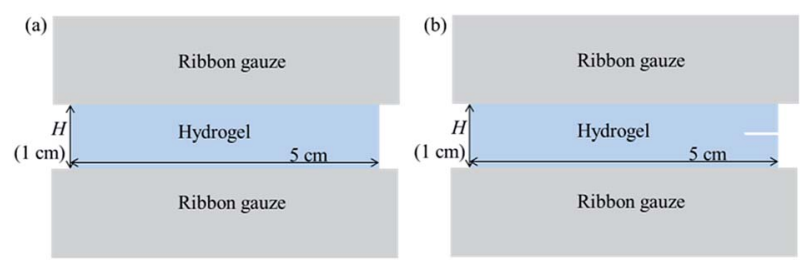

Fig. 1 Schematic of two types of samples for pure shear tests. (a) Sample without a pre-cut crack; (b) sample with a pre-cut crack near the middle of the edge. 
pure shear tests at a loading strain rate of $5 \mathrm{~mm} \mathrm{~min}^{-1}$. In Fig. 2(a) and (b), it is clearly shown that the stress-stretch curves change with different temperatures. With the increase in temperature, the hydrogel undergoes a phase transition: the amount of water in the hydrogel in equilibrium varies with the change in temperature. ${ }^{22}$ Both the maximum nominal stress and maximum stretch increase with the increase in temperature (Fig. 2(c) and (d)). It can be clearly found that the increase in rate of the maximum nominal stress and maximum stretch below the VPTT are smaller than those above the VPTT. Due to non-homogeneous deformation and phase discontinuity, the samples were easily ruptured in a small stretch at $35{ }^{\circ} \mathrm{C}$. The values of maximum nominal stress and maximum stretch at $35{ }^{\circ} \mathrm{C}$ are close to the values below the VPTT. For samples without the pre-cut crack, the maximum nominal stress at $31{ }^{\circ} \mathrm{C}$ is around $5 \mathrm{kPa}$, while the value at $39{ }^{\circ} \mathrm{C}$ is about $25 \mathrm{kPa}$ (Fig. 2(c)). The critical maximum stretch $\left(\lambda_{\mathrm{c}}\right)$ at $39^{\circ} \mathrm{C}$ can be up to 3 , which is twice of the $\lambda_{\mathrm{c}}$ at $31{ }^{\circ} \mathrm{C}$ (Fig. 2(d)). As the NIPA hydrogel is rather sensitive to cracking, the mechanical properties of samples with the pre-cut crack are significantly weaker than the samples without the pre-cut crack in terms of maximum nominal stress and critical stretch (Fig. 2(c) and (d)).

We calculated the Young's modulus $(E)$ from the slope of the stress-stretch curves of samples without the pre-cut crack in $10 \%$ strain by assuming that the NIPA hydrogel is in an elastic state within $10 \%$ deformation. The Young's modulus $(E)$ below the VPTT is about $13 \mathrm{kPa}$, while the Young's modulus $(E)$ above the VPTT is approximately $28 \mathrm{kPa}$. With the increase in temperature through the phase transition, the Young's modulus $(E)$ increases drastically (Fig. 3).

According to the results of Fig. 2, we calculate the fracture energy of the NIPA hydrogel at different temperatures. The calculation method of fracture energy is shown in Fig. 4. The area under the stress-stretch curve gives the strain energy density $W(\lambda)$ (Fig. 4(a)). In the pure shear tests, $W(\lambda)$ is the energy per volume of the uncut samples. The energy release rate $G$ is calculated as follows: ${ }^{23}$

$$
G=H W(\lambda)
$$

(a)

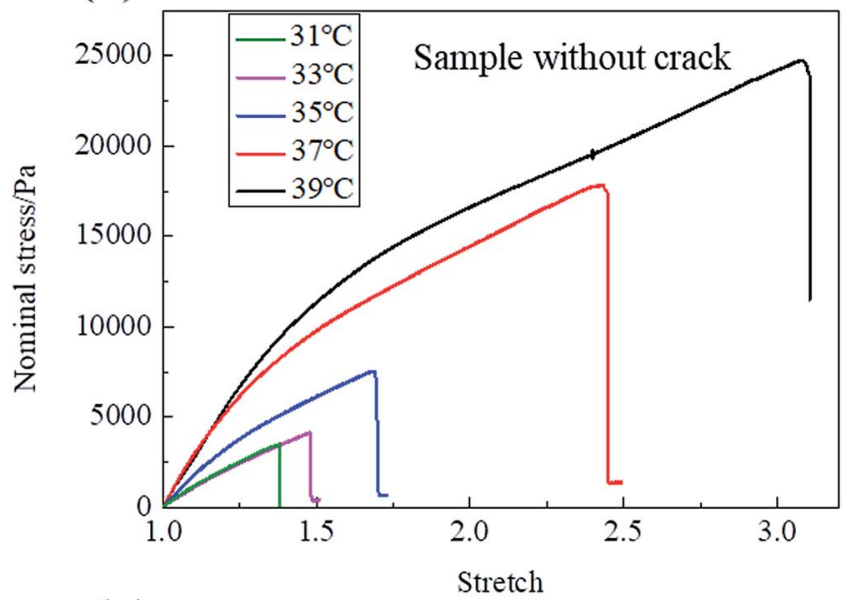

(c)

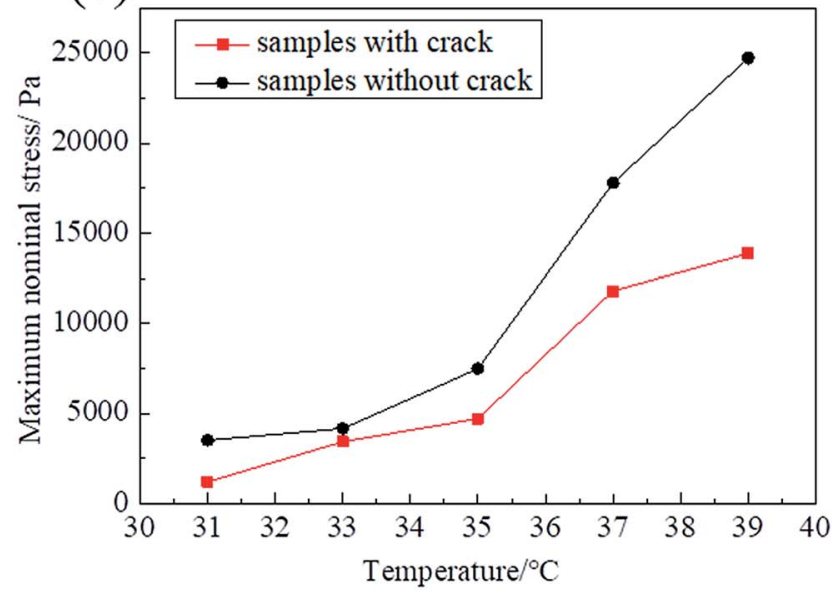

(b)

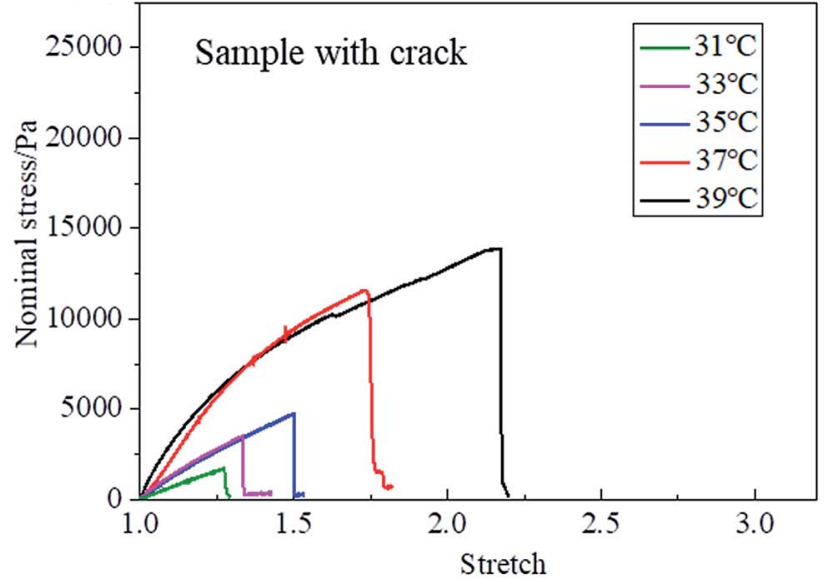

(d)

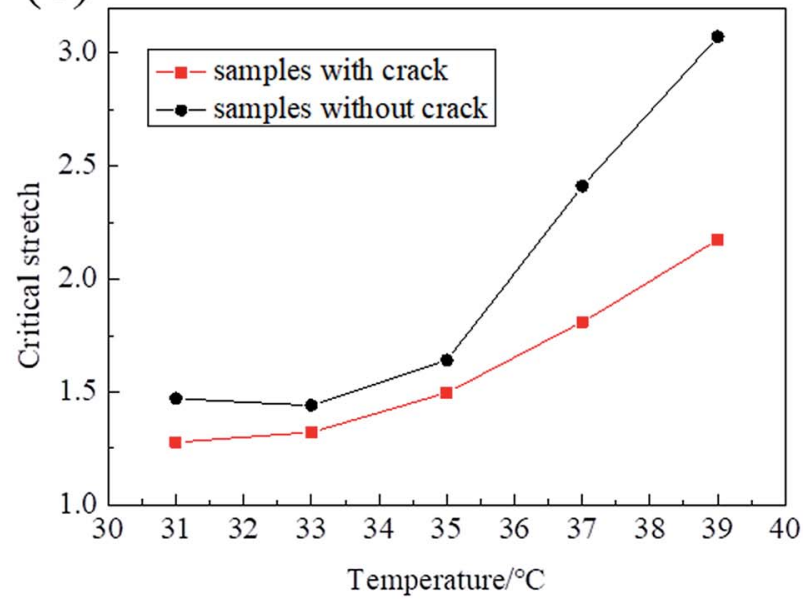

Fig. 2 Fracture characteristics of NIPA hydrogels under monotonic load. (a) Stress-stretch curves for samples without the crack under different temperatures; (b) stress-stretch curves for samples with the crack under different temperatures; (c) the relationship between maximum nominal stress and temperature; and (d) the relationship between critical stretch and temperature. 


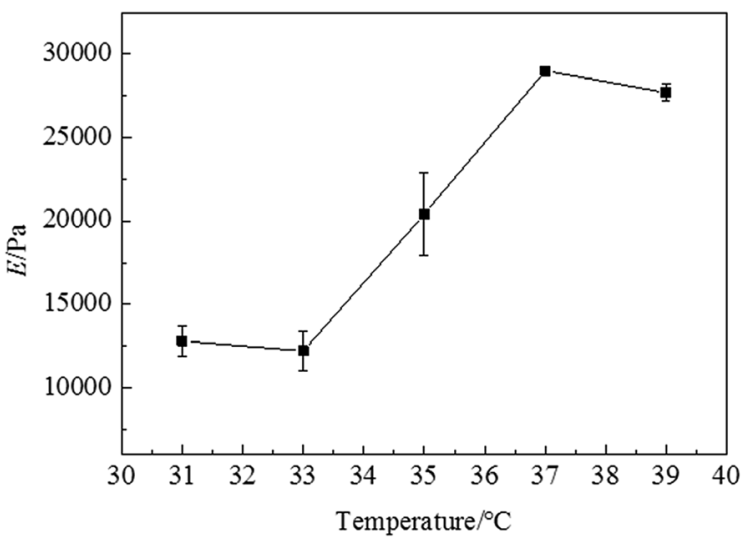

Fig. 3 Young's modulus $(E)$ of the NIPA hydrogels at different temperatures.

where $H$ is the sample length between the fixed bottom and the upper gripper when the sample is undeformed, and $\lambda$ is the vertical stretch. The fracture energy is defined as the critical energy release rate from crack initiation to propagation. Therefore, the fracture energy is calculated from eqn (1) when the stretch $\lambda$ equals the critical stretch $\lambda_{\mathrm{c}}$ of the samples with the pre-cut crack (Fig. 4(b)).

The calculated fracture energy at different temperatures is shown in Fig. 5. The fracture energy of the NIPA hydrogels is approximately $10 \mathrm{~J} \mathrm{~m}^{-2}$ at temperatures below the VPTT. The fracture energy increases rapidly when the temperature is higher than the VPTT. The fracture energy can be up to $150 \mathrm{~J}$ $\mathrm{m}^{-2}$ at $39^{\circ} \mathrm{C}$. In comparison with the existing research results (e.g., the fracture energy of the polyacrylamide hydrogel is around $50 \mathrm{~J} \mathrm{~m}^{-2},{ }^{14}$ the fracture energy of PAMPS/PAAM doublenetwork hydrogels is nearly $3000 \mathrm{~J} \mathrm{~m}^{-2}$ (ref. 15) and the fracture energy of tough hydrogels is close to $10000 \mathrm{~J} \mathrm{~m}^{-2}$ (ref. $24)$ ), the fracture energies of NIPA hydrogels are quite different. This might have originated from the hydrogen bonding and hydrophobicity of the NIPA hydrogels.
The fracture energy increases rapidly when the temperature is higher than the VPTT, mainly because of the intrinsic properties of the NIPA hydrogel. Above the VPTT, the hydrophobic interactions become dominant, and the hydrogel shrinks (or undergoes deswelling) and turns into a collapsed and hydrophobic state expelling water. Therefore, the water content of the hydrogels above the VPTT is lower than that below the VPTT. In ref. 18, it was found that the fracture energy reduces with the increasing in water content. To some extent, the findings in this study further verify the effects of water content on fracture energy.

\section{Fatigue damage}

The fatigue of hydrogels is commonly studied by pure shear tests using samples with/without a pre-cut crack. Bai et al. ${ }^{17}$ defined fatigue damage as the irreversible change in the mechanical property for a sample without a pre-cut crack subject to a cyclic load, while fatigue fracture was defined as the gradual crack propagation for a sample with a pre-cut crack subject to a cyclic load. Herein, we focused on the study of fatigue damage of the NIPA hydrogel. The life of the fatigue damage $\left(N_{\mathrm{f}}\right)$ is defined as the maximum cycles when the nucleates are generated but not completely fractured. The VPTT clearly influences the mechanical properties of the NIPA hydrogels. Therefore, the fatigue damage was studied at temperatures above the VPTT and below the VPTT.

From the information presented in Fig. 2(d), it is observed that the critical stretch increases with the increase in temperature. To improve the efficiency of tests as much as possible, a temperature $\left(37^{\circ} \mathrm{C}\right)$ slightly higher than the VPTT was set to study fatigue damage above the VPTT. According to the loading mode shown in Fig. 6(a), fatigue damage tests were conducted with a prescribed stretch $\left(\lambda_{\max }\right)$ ranging from 1.2 to 1.8 . We loaded the hydrogel without stopping until the crack was observed by the naked eye and recorded the evolution of stressstretch curves over cycles. Fig. 6(b) shows that the maximum nominal stress of each cycle varies with the number of cycles. (a)

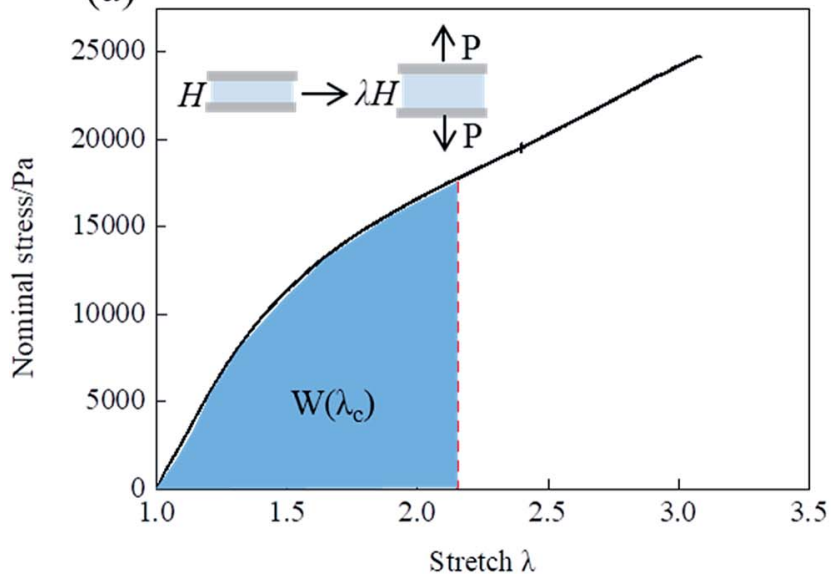

(b)

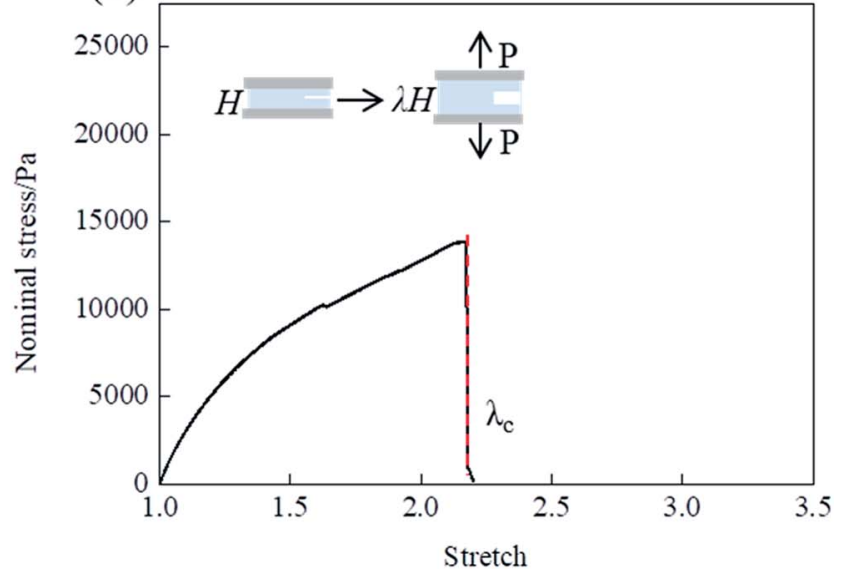

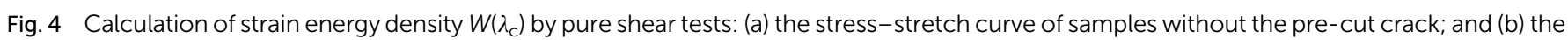
stress-stretch curve of samples with the pre-cut crack. 


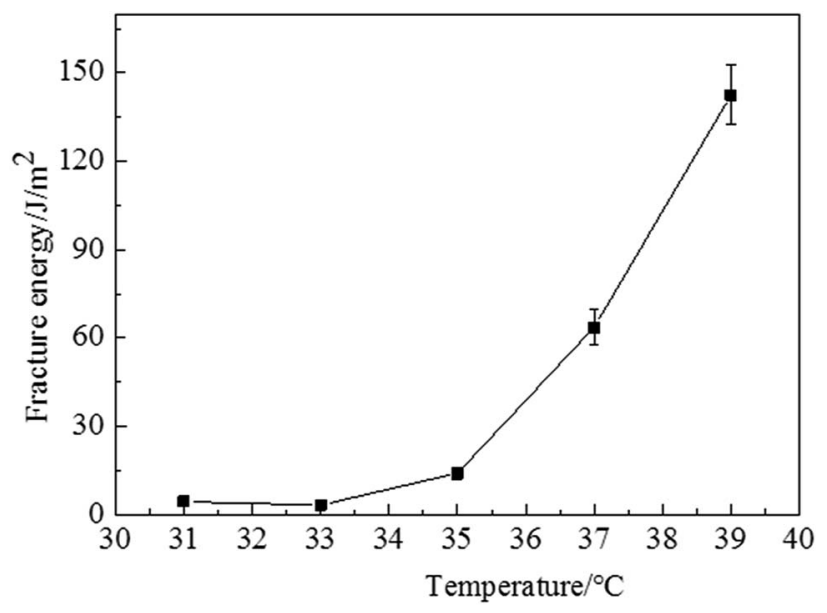

Fig. 5 Fracture energy of the NIPA hydrogels at different temperatures. The data represent the mean \pm standard deviation of three experimental results.

The maximum nominal stress within 1000 cycles reduced rapidly, and then increased with the increase in number of cycles until the generation of nucleates (i.e., the maximum nominal stress suddenly reduced over cycles). The evolution of stress-stretch curves over cycles for NIPA hydrogels still did not reach a relatively steady state, while the robust polyacrylamidealginate hydrogel would reached a steady state after approximately 2000 cycles. ${ }^{16}$ In comparison, the stress-stretch curves for the polyacrylamide hydrogel remained nearly the same with cycling. ${ }^{14}$ Distinct mechanical softening was observed within 1000 cycles due to the breakage of weak bonds and viscoelasticity. The evolution of maximum stress over cycles indicates that the mechanical properties are first weakened and then strengthened over cycles until fatigue damage occurs for the NIPA hydrogels.

As illustrated in Fig. 6(b), the maximum nominal stress becomes 0 shortly after the generation of nucleates for larger maximum stretch cases (i.e., $\lambda_{\max }=1.8,1.7$, and 1.6). This infers that the fatigue propagation life is approximately 0 in comparison with the fatigue damage life $\left(N_{\mathrm{f}}\right)$. For smaller maximum stretch cases (i.e., $\lambda_{\max }=1.2,1.3,1.4$, and 1.5), the maximum nominal stress reduces as number of cycles increases after the peak value. This indicates that the NIPA hydrogel is not very sensitive to nucleates or flaws at a smaller maximum stretch under cyclic loading. Nonetheless, the fatigue propagation life is clearly smaller than the fatigue damage life $\left(N_{\mathrm{f}}\right)$. Therefore, the fatigue life of the NIPA hydrogel is described by the fatigue damage life $\left(N_{\mathrm{f}}\right)$ in this study.

Fatigue damage tests below the VPTT were also conducted using the same loading model as in Fig. 6(a) with a prescribed stretch $\left(\lambda_{\max }\right)$ ranging from 1.1 to 1.27 . A temperature $\left(31^{\circ} \mathrm{C}\right)$ slightly lower than the VPTT was chosen to study fatigue damage for NIPA hydrogels below the VPTT. By observing the recordings of the evolution of stress-stretch curves over cycles, it was found that the evolution features of maximum nominal stress in each cycle over cycles were similar to the evolution features above the VPTT. However, there were several differences on the stress-stretch curves because the responses of the polymer network under a cyclic load at different temperatures were different. ${ }^{20}$ Although the hysteresis between the loading and unloading curves is generated due to the damage of the polymers network, the hysteresis loop is clearly smaller than that above the VPTT. Furthermore, the hysteresis loop in the first cycle is mostly identical to that of subsequent cycles. Residual strain is also observed at the end of the initial hundreds of cycles. After hundreds of cycles, the residual strain completely disappears as the stress is larger than 0 when the stretch returns to 1 for smaller $\lambda_{\max }$ cases.

The number of cycles $\left(N_{\mathrm{f}}\right)$ corresponding to the maximum nominal stress for different $\lambda_{\max }$ cases was obtained, as shown in Fig. 6(b). The experimental data is shown using black dots and triangles in Fig. 7. It is clearly observed that the fatigue life under the same stretch at $37^{\circ} \mathrm{C}$ is larger than that at $31^{\circ} \mathrm{C}$. It seems that $N_{\mathrm{f}}$ is exponentially reduced with the increase in stretch. The experimental data were fitted using the following equation:

$$
\lambda=\lambda_{\mathrm{f}}+A_{0} \mathrm{e}^{-N_{\mathrm{f}} / B_{0}}
$$

(a)

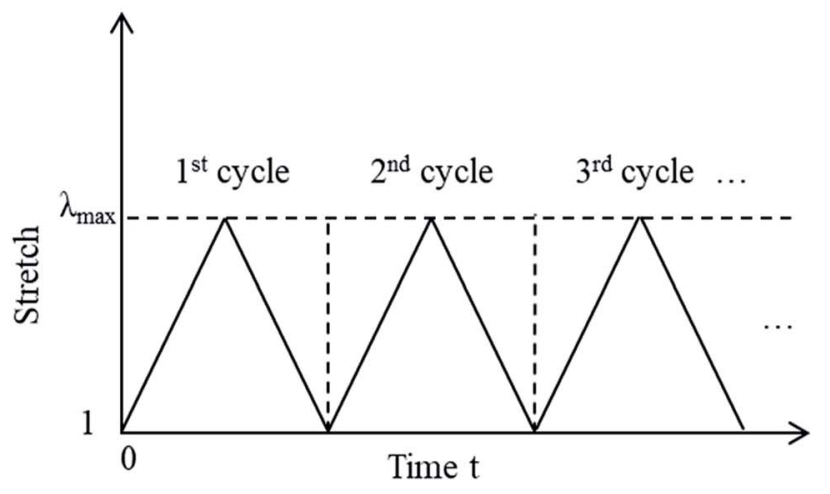

(b)

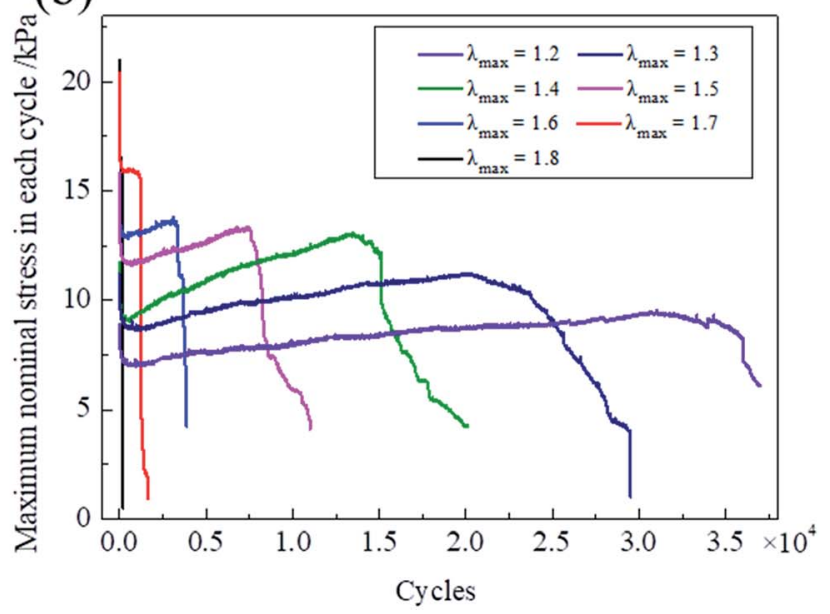

Fig. 6 The loading mode under cyclic load (a) and the maximum nominal stress measured with cycles at $37^{\circ} \mathrm{C}$ (b). 


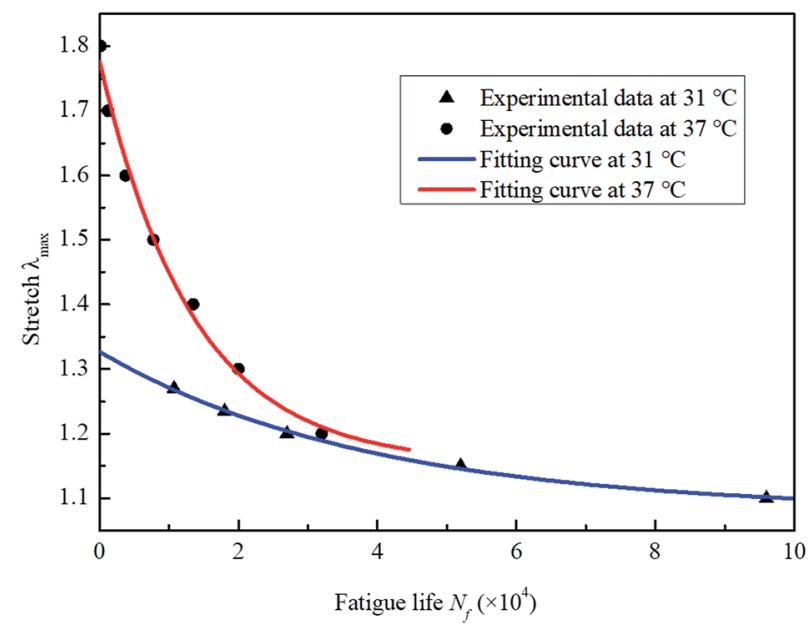

Fig. 7 The relationship of $\lambda-N_{f}$ for the NIPA hydrogel at $31^{\circ} \mathrm{C}$ and $37^{\circ} \mathrm{C}$.

where $\lambda$ is stretch, $\lambda_{\mathrm{f}}$ is fatigue limit, $N_{\mathrm{f}}$ is fatigue life, and $A_{0}$ and $B_{0}$ are the two fitting parameters.

The fitting curves are shown in Fig. 7 in red and blue for the cases of $37^{\circ} \mathrm{C}$ and $31^{\circ} \mathrm{C}$, respectively. It seems that the fitting curve agrees well with the experimental data. The fitting parameters are $\lambda_{\mathrm{f}}=1.15, A_{0}=0.624$ and $B_{0}=1.343 \times 10^{4}$ for the case of $37^{\circ} \mathrm{C}$, while they are $\lambda_{\mathrm{f}}=1.08, A_{0}=0.247$ and $B_{0}=3.904$ $\times 10^{4}$ for the case of $31^{\circ} \mathrm{C}$.

By analysis of the fatigue life of the NIPA hydrogels at different temperatures, it was found that the fatigue life should be in the order of magnitude of 4 . By comparison, the fatigue life of rubber should be approximately $107 . \lambda_{\mathrm{f}}$ varies with temperature. It was found that $\lambda_{\mathrm{f}}$ above the VPTT is larger than that below the VPTT. The fatigue life above the VPTT is also larger than that below the VPTT. Hence, it is noted that the fatigue limit $\lambda_{\mathrm{f}}$ should be determined by the lowest temperature to prevent the NIPA hydrogels from fracturing during the fatigue life.

\section{Conclusions}

By pure shear tests, the fracture behaviours and fatigue damage of the NIPA hydrogels at different temperatures were obtained. The fracture energy measured under monotonic load increases with the increase in temperature. Moreover, the fracture energy is approximately $10 \mathrm{~J} \mathrm{~m}^{-2}$ below the VPTT, while the fracture energy is up to $150 \mathrm{~J} \mathrm{~m}^{-2}$ at $39^{\circ} \mathrm{C}$. The analysis of the evolution of stress-stretch curves over numerous cycles indicates that the mechanical properties of the NIPA hydrogel under cyclic load are first weakened and then strengthened until fatigue damage occurs. The fatigue life $\left(N_{\mathrm{f}}\right)$ increased with the increase in temperature. By fitting the experimental data using the equation $\lambda=\lambda_{\mathrm{f}}+A_{0} \mathrm{e}^{-N_{\mathrm{f}} / B_{0}}$, it was found that the fitting curve agreed well with the experimental data as the fatigue limit $\lambda_{\mathrm{f}}$ was 1.15 and 1.08 at $37{ }^{\circ} \mathrm{C}$ and $31{ }^{\circ} \mathrm{C}$, respectively. The fatigue limit $\left(\lambda_{\mathrm{f}}\right)$ should be determined by the lowest temperature to prevent the NIPA hydrogels from fracturing during the fatigue life due to the variation in fatigue limit $\left(\lambda_{\mathrm{f}}\right)$ with temperature. The findings in this study might be important for the engineering applications of NIPA hydrogels.

\section{Conflicts of interest}

There are no conflicts to declare.

\section{Acknowledgements}

This study was supported by the National Natural Science Foundation of China (11372236 and 11572236).

\section{Notes and references}

$1 \mathrm{~J}$. P. Gong, Why are double network hydrogels so tough?, Soft Matter, 2010, 6(12), 2583-2590.

2 Z. S. Liu, W. Toh and T. Y. Ng, Advances in Mechanics of Soft Materials: A Review of Large Deformation Behavior of Hydrogels, Int. J. Appl. Mech., 2015, 7, 1530001.

3 C. M. Hassan and N. A. Peppas, Structure and applications of poly (vinyl alcohol) hydrogels produced by conventional crosslinking or by freezing/thawing methods, in Biopolymers.PVA Hydrogels, Anionic Polymerisation Nanocomposites, Springer, 2000, pp. 37-65.

$4 \mathrm{~K}$. Y. Lee and D. J. Mooney, Hydrogels for tissue engineering, Chem. Rev., 2001, 101(7), 1869-1880.

5 P. S. Lienemann, M. P. Lutolf and M. Ehrbar, Biomimetic hydrogels for controlled biomolecule delivery to augment bone regeneration, Adv. Drug Delivery Rev., 2012, 64(12), 1078-1089.

6 J. W. Jeong, M. K. Kim, H. Cheng, W. H. Yeo, X. Huang, Y. Liu, Y. Zhang, Y. Huang and J. A. Rogers, Capacitive Epidermal Electronics for Electrically Safe, Long-Term Electrophysiological Measurements, Adv. Healthcare Mater., 2014, 3(5), 642-648.

7 J. Li, A. D. Celiz, J. Yang, Q. Yang, I. Wamala, W. Whyte, B. R. Seo, N. V. Vasilyev, J. J. Vlassak, Z. Suo and D. J. Mooney, Tough adhesives for diverse wet surfaces, Science, 2017, 357(6349), 378-381.

8 Y. R. Zhang, L. Q. Tang, B. X. Xie, Z. J. Xu, K. J. Liu, Y. P. Liu, Z. Y. Jiang and S. B. Dong, Int. J. Appl. Mech., 2017, 9, 1750044.

9 R. Langer, Drug delivery and targeting, Nature, 1998, 392(6679), 5-10.

10 P. Ferreira, J. Almeida, J. Coelho and M. Gil, Photocrosslinkable Polymers for Biomedical Applications, INTECH Open Access Publisher, 2011.

11 S. J. Zheng, Z. Q. Li and Z. S. Liu, The fast homogeneous diffusion of hydrogel under different stimuli, Int. J. Mech. Sci., 2018, 137, 263-270.

12 S. Xu, Y. Wang, J. Y. Hu and Z. S. Liu, Int. J. Appl. Mech., 2016, $8,1640002$.

13 J. H. Wu, P. F. Li, C. L. Dong, H. T. Jiang, B. Xue, X. Gao, M. Qin, W. Wang, B. Chen and Y. Cao, Rationally designed synthetic protein hydrogels with predictable mechanical properties, Nat. Commun., 2018, 9(620), 1-11. 
14 J. Tang, J. Li, J. J. Vlassak and Z. G. Suo, Fatigue fracture of hydrogels, Extreme Mech. Lett., 2017, 10, 24-31.

15 R. B. Bai, Q. Yang, J. Tang, X. P. Morelle, J. Vlassak and Z. Suo, Fatigue fracture of tough hydrogels, Extreme Mech Lett., 2017, 15, 91-96.

16 W. L. Zhang, X. Liu, J. K. Wang, J. D. Tang, J. Hu, T. Q. Lu and Z. G. Suo, Fatigue of Double-Network Hydrogels, Eng. Fract. Mech., 2018, 187, 74-93.

17 R. B. Bai, J. W. Yang, X. P. Morelle, C. H. Yang and Z. G. Suo, Fatigue Fracture of Self-Recovery Hydrogels, ACS Macro Lett., 2018, 7, 312-317.

18 E. R. Zhang, R. B. Bai, X. P. Morelle and Z. G. Suo, Fatigue fracture of nearly elastic hydrogels, Soft Matter, 2018, 14, 3563-3571.

19 S. J. Zheng and Z. S. Liu, Phase transition of temperature sensitive hydrogel under mechanical constraint, J. Appl. Mech., 2018, 85, 021002.
20 H. G. Schild, Poly(N-isopropylacrylamide): experiment, theory and application, Prog. Polym. Sci., 1992, 17, 163-249.

21 A. Suzuki and T. Ishii, Phase coexistence of neutral polymer gels under mechanical constraint, J. Chem. Phys., 1999, 110(4), 2289-2296.

22 N. Zhang, S. J. Zheng, Z. Z. Pan and Z. S. Liu, Phase Transition Effects on Mechanical Properties of NIPA Hydrogel, Polymers, 2018, 10(358), 1-11.

23 R. S. Rivlin and A. G. Thomas, Rupture of rubber. I. Characteristic energy for tearing, J. Polym. Sci., 1953, 10(3), 291-318.

24 J. Y. Sun, X. H. Zhao, W. R. K. Illeperuma, O. Chaudhuri, K. H. Oh, D. J. Mooney, J. J. Vlassak and Z. G. Suo, Highly stretchable and tough hydrogels, Nature, 2012, 489, 133-136. 